\title{
How to achieve a unified theory of information
}

\author{
Wolfgang Hofkirchner
}

Center for Advanced Studies and Research in Information and Communication Technologies and Society, Paris Lodron University of Salzburg, Austria; e-mail: wolfgang. hofkirchner@sbg.ac.at

\begin{abstract}
The paper deals with the necessity and feasibility of an integrated information theory. It develops guidelines for how to conceive of information in a way that avoids the pitfalls of certain ways of thinking like reductionism, projectivism or disjunctivism.
\end{abstract}

Keywords: Information Society, Two Cultures, Subject-Object-Dialectic, Ways of Thinking

1 t was about 10 years ago when I helped organise the second conference on the Foundations of Information Science in Vienna and published proceedings with the title "The Quest for a Unifying Theory of Information". While a considerable number of scientists still today disbelieve in the feasibility of a single generic concept of information, there are several attempts to hypothesise or theorise information in a unifying manner carried out by a strong minority of scientists. E.g., a question put forward by Hans von Baeyer to the audience at the last International Conference on Foundations of Information Science held in Paris in 2005 showed a fifty-fifty vote for either option.

The skeptics are right in problematising the threat of being subject to dogmatism. The camp of "unifiers" are right when being unsatisfied by a fragmented world picture. Is there a way to avoid both dogmatism and fragmentation?

The paper attempts to argue for a positive answer. It starts from the necessity of an integrated information theory for reasons of finding a way out of the current crisis of civilisation and then shows that an integrated theory is feasible. First it lists several concepts different from the information concept and argues that their relation to the information concept be taken into consideration. It gives an account of possible classifications of existing information concepts and theories. After that it develops a perspective from which integration can be achieved without doing harm to any of the ideas in question. This perspective is the perspective of unity-through-diversity. Ways of thinking, in particular, reductionism, projectivism, disjunctivism and integrativism are clearly defined in order to yield guidelines for how to conceive of information.

\section{A Unified Theory of Information (UTI) - What for?}

At first glance, it seems an intrascientific issue of whether or not in the field of information there is an attempt to grasp the big picture and develop a shared theory by which the whole variety of different manifestations of information processes in society and in the world at all might be understood. Like in everyday's thinking where people strive for connecting unconnected experiences and even reconciling irreconcilable experiences in order to arrive at a coherent overall view (just think of the psychologically well-described tendency of ordinary people to avoid cognitive dissonance), science is heading for consilience -a term attracting interest when Edward $\mathrm{O}$. Wilson published his book of the same title (1998)-, that is, a unity of knowledge, that 
allows for better and better explanations and predictions. This is accomplished via the construction of new theories that include the findings of the old theories as kind of approximations and at the same time are able to explain and predict phenomena that were not covered by the old theories. Thus, in science there is a tendency towards more and more overarching theories, towards more and more generalising theories, towards more and more universal theories. Unified theories address the universal by unifying the multiplicity of so far incoherent theories bound to particular levels. Unified theories belong to the intrascientifc progress towards the universal.

However, it is not just a case of pure scientifc curiosity. If we take into acount that science is not work in an ivory tower but a social undertaking that satisfies social demands, that is, that there is an extrascientific function all science has to fulfil - the betterment of social life and solving problems that arise from social practice -, then it does not come as a surprise that on the threshold of the information age science is concerned with information and that there is a quest for a unified theory of information (UTI) (see Hofkirchner 1999).

The information age is the age of information societies which industrialised societies are transforming into which is visible by the spread of new information and communication technologies (ICTs), while the industrial age is the age of industrial societies into which agricultural societies have been transforming worldwide. Each transformation is known as a revolution and all revolutions together are said to form the evolution of civilisation (see Fig. 1).

On the one hand, there is a lag of scientific development behind societal and technological development. Development in technology is not accompanied by an equally rapid growth in scientific insight, let alone foresight, as to the impacts of technology on levels of society other than that of technological organisation. Attempts to observe and understand the basic nature of this change are still second place. The public use of the notion of "information society" has been reduced to denoting a society in which applications of modern ICT are widely spread in order to facilitate the handling of what commonly is called "information". A scientific understanding of this transformation has not had time to develop. There is not yet a proper "science of the information society" or a proper "science of information".

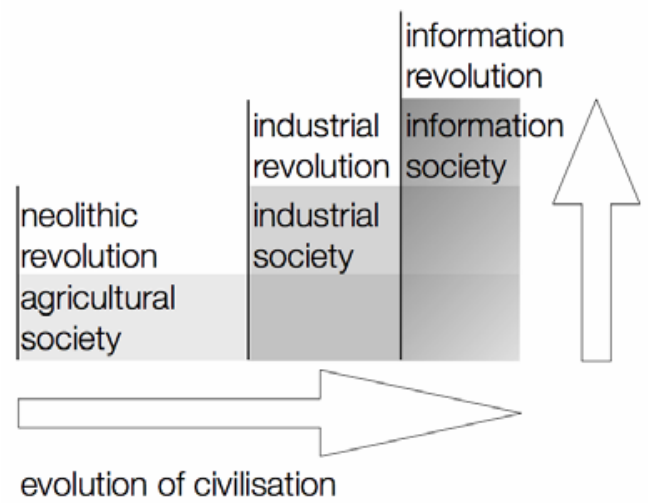

Figure 1: From industrial societies to information societies

On the other hand, the state of the relationship between science and technosocial development of today regarding information can e.g. be compared to the state Karl Marx was confronted with in respect to labour. In his time labour could become and necessarily became a matter of scientific interest, since labour as a matter of fact had gained a new role in society. It became something more abstract in social life, that is, it was treated in society irrespective of its concrete characteristics. Marx called that a "real-abstraction" -an abstraction that occurred in reality due to the real treatment of labour in emerging capitalism which became the basis for the general concept of labour in scientific thought. It was only then that the concept of labour could be stretched back to former social life in the history of humanity and that other phenomena than industrial work could be subsumed under the concept of labour, albeit as different manifestations. Making use of this notion of realabstraction we might assume that information has gained as decisive a role in society nowadays so as to foster a new scientific conceiving and theorising - that it has turned into a real-abstraction which is the rationale for devising a general idea as well: what labour is in regard to human history as seen from the perspective of industrial society, information is in regard to history from the perspective of information society. 
What then has changed with information? Is it just the quantity of what can be conveyed by ICTs? Or is the quantity of that just the indication for a qualitative change taking place?

There has been a qualitative change in the role information can play for the development of society, and this change is unprecedented in the history of humanity. Information has become the bearer of survival, the key to our future. For the information age is, fundamentally, the age of global challenges. The impressions made by the atomic bomb, industrial and agricultural catastrophes, hunger, suffering and death in the poor parts of the world, starting in the middle of the last century but persistent in the new millennium, have raised consciousness of the destructive and fallible nature of the human technosphere, the fragile and finite nature of the human ecosphere, and the unsettled, unbalanced nature of the human sociosphere. It has become a part of general knowledge to realise that the existence of such global challenges can endanger the persistence of today's societies all over the world. The global problems are global in a twofold sense: first, they concern humankind as a whole (as object); second, they can also only be solved by humankind as a whole (as subject). The risk this crisis carries is that humankind may be wiped out. The chance it offers, however, is that humankind may be raised to another level of humanity. It is disparities in the development of the relations amongst humans, between humans and nature and between humans and technology that build obstacles to keeping society as a whole on a stable, steady path of development. It is malfunctions in the sociosphere, ecosphere and technosphere that continue to aggravate the global challenges. And it is information that turns out the only remedy. It is information that is required to steer society. It is information that is required to reorganise humanity onto a higher level of organisation. It is information that is required to alleviate and reduce the frictions (see Heylighen 2007) in the functioning of those systems that make up humanity from the individual to ethnicities to nations to world society; from economy to politics to culture; from society to ecology to technology; from the social realm to the biotic realm to the physical realm. In a word, the continued existence of humanity has shaped up as impossible without conscious and cautious intervention in the process of its own development including all spheres of intervention. This intervention that orients towards the relinking of our world falling apart due to processes of heterogenisation, fragmentation and disintegration is informational in its nature, but as it extends from the human to the living to matter, it necessitates a deep understanding of the information processes going on in all the world we inhabit.

Knowledge as capacity to act means today the capacity to act vís-a-vís the global challenges means knowledge about how information guides the processes that puts us at risk. Hence information is the conditio sine qua non for the further existence and development of humanity.

From this perspective, a UTI makes sense.

\section{What is the Extension of the Con- cept of Information in a UTI?}

"Information" is the superconcept, it is a generic concept. It covers all different manifestations of real-world information processes regardless of the realm in which they appear.

It is clear that "information" is closely related to a bunch of similar concepts. The choice of one out of them and to illuminate how it is linked to the others is kind of arbitrary. It is rather a terminological issue. What matters is the intension of the concept, that is, what it means and how the network of relations is conceived of.

Here is an incomplete list of concepts that are related to the superconcept of "information" and are -to a major or minor extentcomprised by it, that is, they are -in different degrees- overlapping with "information":

- "structure"

- "data"

- "signal"

- "message"

- "signification", "meaning", "sense"

- "sign"

- "sign process", "semiosis"

- "psyche"

- "intelligence"

- "perception" 
- "thought"

- "language"

- "knowledge"

- "consciousness", "mind"

- "wisdom"

- ...

The scope of a UTI is thus as far and deep as that.

For practical reasons, it makes sense to make use of the following distinction I introduced elsewhere (Hofkirchner 2002, see also Hofkirchner/Stockinger, 2003).

We come across information in three areas of society:

- in the area of cognition, that is, where the contents of consciousness is produced by individuals,

- in the area of communication, that is, where common understanding is produced by interactions (individuals),

- in the area of co-operation, that is, where sense embodied in societal structures is produced collectively by individuals who act in balanced ways.

The first and second areas go without saying, with cognitive science and communication studies as well-known fields of scientific activity. It is the third area that proves unconventional, as it contests a strong tradition in humanities that qualifies society as composed of communications only. Niklas Luhmann stands for this tradition. Introducing co-operation does, actually, justice to the "social facts" Emile Durkheim considered the proper object of sociology, to the "social relationships" Karl Marx distinguished from "social behaviour", to the "structure" that was focused on by the structuralist school after Marx, to the "synergy effects" that today can be investigated by science-of-complexity methods. That is, it does justice to the phenomenon that there is more to society than only communication on the level of interaction of individuals and that this whole - which is more than the sum of communications/interactions - is an information process too, albeit on the level of a social group.

Hence we can say, a UTI comprises human cognition processes, human communication processes, and human co-operation processes. All three of them are, in a way, norma- tive: cognition has the objective to position the individual vís-a-vís the societal, social, and nonhuman environment; communication aims at finding a state of mutual understanding between individuals on whatever matter; and co-operation has a goal - that of a state of organisation of individuals that allows for a mutually beneficial common outcome. As a consequence, cognitive science, communication studies, and cultural studies, social science, humanities, arts and the like insofar as dealing with the added value are sciences that inquire into human information processes.

But "information" is not a concept that applies to humans only. A UTI has to apply it to the precursors of human information processes as well. Cognition is not only a process on the human level, you will find it with other organisms as well. The same holds for communication. And for co-operation, too. Furthermore, it depends on the intension of the "information" concept whether or not also precursors of organismic cognitive, communicative and co-operative information processes can be identified in the prebiotic world.

\section{Which are the Theories/Concepts of Information that a UTI attempts to unify?}

There are several possible classifications at hand.

\subsection{A philosophical classification}

The oldest way to classify information concepts/theories - which has a long history back in philosophy- is to inquire for the essence of information, for the nature of information, for the substance out of which it is made up. This is a question which is answered in relation to the essence, nature, and substance of matter.

The first answer is that information is of the same substance as matter. Either this substance is conceived as something material and then information is something material. This answer is material(istic) monism: everything is like matter and so does information. That's called materialism.

Or this substance is said to be immaterial and then information is something immaterial. This answer is immaterial (ideal, idealistic, ideational, informational) monism, idealism: 
also matter is like mind (information). Varieties are Platonism and Radical Constructivism.

Another answer is that matter and information do not share the substance: they are essentially different in nature. Matter is material and information is not: this is the answer of dualism. Here another question arises: are these two substances inert and no reactants to each other or do they interact and, if so, how then can one side of the duality affect the other side? How is it possible that matter influences mind (information)? How is it possible that mind (information) be efficacious on matter? Suffice it to mention the Cartesian tradition and more recently John Eccles who tried to give an answer together with Karl Raimund Popper (1977).

Since this classification is a philosophical one, it belongs to the most abstract classifications.

\subsection{A disciplinary classification}

Broken down from philosophy to an account of the disciplines, there is the gap between the two cultures of (natural) science and social and human sciences that has to be considered in approaching information - a gap between the natural and the engineering sciences (including formal sciences) on the one hand and the arts and humanities (including the social sciences) on the other hand that dates back to the 17th century and to philosophers such as, again, René Descartes. The gap between the two branches in science reached its heights in the late 19th century with the works of Neo-Kantian philosophers, scientists, and literary intellectuals such as Wilhelm Windelband and Heinrich Rickert. Wilhelm Windelband (1894) for example introduced the disjunction between "nomothetic" (meaning: the law) and "ideographic" (meaning: the event), which would remain to exist alongside one another as the final, incommensurable forms of our notions about the world. Today this cleft is known as C. P. Snow's dilemma which he bemoaned in 1959 and 1963 (see e.g. 1998).

The science and technology side of this cleft is characterised by a technologically bounded rationality which rests upon the obsolete equation of social and scientifictechnological progress. The second is charac- terised by a humanistic rationality which is ignorant of the field of science and technology. So are the categories of information concepts.

The first approach is inclined to be reductionistic by method. It reduces different qualities of the phenomena under investigation to one and the same quality which is the most simple as a rule. It can be said it looks upon information as something that can be received, stored, processed, exchanged, used, and so on, as if a thing. It is the "hard" science's standpoint. This shall hold for cognition, communication and co-operation processes in society and for natural domains as well.

The second approach is biased, insofar as it takes as point of departure the stance of humanities. Methodologically, there are two possibilities. Either it projects one particular quality in question which, as a rule, is the most complex one onto phenomena which do not possess this quality, and pretends to be able to discover them there. Properties of information in nonhuman domains are usually extrapolated from properties of information in the human domain (anthropo(socio)morphism). Beyond that, properties of cognition may be extrapolated from those of communication, and those of communication, in turn, from those of co-operation within the human domain itself.

Or the attempt at a subsuming, though unifying, solution is given up and it is argued in favour of a lack of comparability of the given phenomena in nature and society. In this dichotomising view information is exclusively ascribed to the human domain. Beyond that, it may exclusively be ascribed to particular incidences within the human domain.

In both humanities-oriented cases, information is basically considered a human construction. It is the stance of so-called "soft" science.

\subsection{A clusters classification}

The most concrete classification might be along certain clusters of common perspectives.

A first cluster of information concepts/theories - wherever you want to begin with - might be those that look upon information as a given. Sometimes this is called "po- 
tential" information or "structural" information. It is structural sciences that deal with that topic. According to them, matter is always in a certain shape, gestalt, form, and this form is information (Bernd-Olaf Küppers is a prominent advocate of this position which is espoused with the notion of "Strukturwissenschaften" - "Structural Sciences" - introduced in the 70ies of the last century by Carl Friedrich von Weizsäcker, see Küppers 2000).

A second cluster focuses on the transmission aspect. Seen from that angle, information is not lying in the structure but that which is transmitted from a sender to a receiver via a channel that is disturbed by noise. That is the classical view inaugurated by Shannon and Weaver which is considered the mother of all communication models (see the original article by Shannon 1948). What is said here to flow, to float, is sometimes called "free" information.

A third and last cluster is that of the view of the receiver. Information is finally not that which is transmitted - pursuant to this perspective - but that which is processed by the receiver. It is the receiver who, by processes of decoding, is considered to attach a meaning to the message and to thereby produce "actual" information. This is the leitmotif of all developments in communication studies, in particular, cultural studies, that tried to complement, or depart from, the channel model.

So the range of theories or concepts of information (and related phenomena) that from a UTI point of view are to be subjected to an attempt of unification - is as wide as shown in the three classifications presented here.

\section{How can unification be achieved?}

As a start, the review of the classifications of information concepts/theories so far seems to support the assumption of a multitude of approaches that are diverse and irreconcilable and do not offer the possibility of consolidation. But on closer scrutiny, no matter where you start from - either philosophy or the two cultures or the disciplinary point of departure , you will end up with one and the same scheme. For climbing down the ladder from philosophy to scientific disciplines, the categorisation of the existing information con- cepts/theories seems like a concretisation and specification of the rather abstract and unificational classification in that the rather down-toearth classes are embedded in the rather lofty classes. The clusters are embedded in the scientific cultures and the scientific cultures are embedded in philosophy. It is not between the levels but just at each level that there is some discrepancy that forms an obstacle to unification.

According to the philosophical classification, information concepts are stuck between materialism and idealism. Given the "hard""soft" science divide, information concepts/theories are stuck between the "hard" and "soft" side. But it does not come as a surprise that the "hard" side is materialistic as to the philosophical inclination and the "soft" side idealistic. And as to the clusters, too, "information" seems to be exclusively "potential" or exclusively "free" or exclusively "actual". But the first and the second notion belong to the science and technology ("hard") side of the scientific divide, while the third notion has an affinity to the humanities ("soft") side.

How can this basic divide be successfully bridged? The answer is like in the story of the elephant and the blind men (or the men in a dark room) each of whom touches a different part of the elephant and mistakes the part for the whole (Saxe, 2003). So none of the various existing information concepts/theories should take its perspective absolute but, in a way, complementary to the other perspectives.

But how can matter and idea, mind, information, be grasped as complements - and with them information as dealt with as a thing (a structure, a flow) or as human construction (a processing activity)?

Taking into account that philosophy is not only about the essence or the nature or the substance of reality - which is ontology - but also about praxis - which is praxiology, including, in my view, ethics and aesthetics and about the empirical - which is epistemology and methodology -, and assuming that praxiology, ontology and epistemology form a kind of hierarchy (a Praxio-onto-epistemology I introduced elsewhere, see Hofkirchner et al. 2005 ) with the praxic point of view prior to the 
ontic point of view prior to the epistemic one, we can ask whether or not the perspective of praxis provides us with a still more abstract view of information than the materialismidealism divide already did and we can ask to what extent this view can help us with unification through reworking and reinterpreting the classifications.

Now, in praxiology the point is to look upon everything in terms of objects and subjects and the relationships between them. Objects and subjects are defined by mutual exclusion. Objects are subject to subjects, subjects subject objects. Humans are subjects. Through interference with their human and non-human surroundings they produce objects. These objects tend to "object" to becoming subject to humans as there is inertia with them. Praxis is the ongoing process of subjecting objects to humans while factoring in inertia. Objects do not exist, unless subjects exist, and vice versa, and they are bound together by the process and relationship of praxis. This relationship is known as a dialectical one; a dialectical relationship is said to exist, if the following criteria come true: firstly, both sides of the relation are opposed to each other; secondly, they depend on each other; thirdly, they are asymmetrical in that neither side can be replaced with the other without simultaneously replacing the mode of relationship. Masterand-slave or mother-and-daughter are examples for dialectical relationships.

Now we recognise: the information concept/theory classifications presented above depend on how they view the object-subjectrelation.

According to the materialistic (as regards philosophy), "hard" science (as regards the two cultures), and structuralistic and communication stance (as regards the disciplines), we find that information is something objective - it seems not to belong to a subject - that can be measured independently. And according to the idealistic, "soft" science and recipient's view, information is considered something subjective, that is, inextricably linked to a subject that is human.

A UTI cannot be satisfied by such onesided views. An integrative information science has to consider both the objective and subjective aspects of information and overcome objectivism and subjectivism as well.

The objectivist outlook is right in stating that information is a phenomenon out there and not merely human imagination. The subjectivist outlook is right insofar it states that information occurs only if there is freedom of choice for that to which generating and disposing of information is attributed. However, regarding the objectivist outlook, we have to limit the scope of objects with which information is said to be found to those objects exclusively that take the role of subjects, and regarding the subjectivist outlook, we have to enlarge the sphere of subjects from that of humans exclusively and include non-human ones, too.

Since the materialism-idealism divide can be derived from the object-subject divide in that matter is objective and ideas are subjective, it might become clear that matter and ideas belong together as objects and subjects do.

An answer that goes beyond (materialistic and idealistic) monism and (if you like, interactive) dualism is the answer of dialectics. Dialectics recognises identity and difference of matter and information at the same time. It recognises identity, given the difference, for this identity makes it possible that these different sides interact. And it recognises the difference, given identity, for this makes it possible to differentiate matter and information as different specifications of an identical, common, genus. So in Emergentist Materialism - which is an example for this answer matter is, so to say, the common substance but leaves room for emergent properties and events like mind (information) which is of a different materiality compared with the simple, pure, materiality that occurs in the nonemergent state of matter (see Bunge 1980).

An answer that goes beyond the divide of the two cultures is the answer of a "third" culture. Snow envisioned this third culture with the words: "With good fortune, however, we can educate a large proportion of our better minds so that they are not ignorant of imaginative experience, both in the arts and in science, nor ignorant either of the endowments of applied science, of the remediable suffering of most of their fellow humans, and of the 
responsibilities which, once they are seen, cannot be denied" (Snow 1998, 100). John Brockman, a US-American publisher and author, refers to Snow. According to Brockman, the third culture is "founded on the realization of the import of complexity, of evolution. Very complex systems - whether organisms, brains, the biosphere, or the universe itself were not constructed by design: all have evolved" (Brockman 1995, 20-21). So information processes originated from evolution and underwent evolution from early, rudimentary forms to advanced forms we face today. Social science is the discontinuous continuation of natural science inasmuch as the social forms of information processes are the discontinuous continuation of natural forms of information processes.

An answer that goes beyond the particularisation of the disciplinary clusters is the answer that the third kind of categorisation turns out to be alongside steps of information processes (processing). Altogether, the three clustered perspectives seem to give a picture of a series of steps of information processes. The first step - information frozen to a structure - seems to represent something that might enter the information process. The second step is then the leaking of melted and liquified information, the reaching out of the "potential" information just by virtue of its showing up to whatever there is out. And the third step - the "actualisation" of the "potential" information by an agency - could be seen as a step in which the process is frozen down again, but finds itself in another structure, in a new structure of this very agency (which, in turn, as new "potential" information might become a new starting point). In separation, however, these aspects can account for a fragmented picture only.

So the concept of information in a UTI is a concept that leaves the subject/object divide behind. It is a concept that is objectve and subjective at the same time.

\subsection{What is the meaning of Capurro's Trilemma with regard to the at- tempts of unification?}

Capurro's Trilemma runs like this (Capurro et al. 1997): in attempting to define and determine what "information" means throughout the disciplines (as well as in everyday thinking) and what it should or could mean you are facing a logical situation that offers three options none of which, however, is satisfactory (see Table 1).

Table 1: Capurro's Trilemma

\begin{tabular}{|l|l|}
\hline $\begin{array}{l}\text { information } \\
\text { concepts }\end{array}$ & $\begin{array}{l}\text { relationship between intension (meaning) and } \\
\text { extension (fields) }\end{array}$ \\
\hline synonymity & $\begin{array}{l}\text { there is one singular meaning that applies to every } \\
\text { field }\end{array}$ \\
\hline analogy & $\begin{array}{l}\text { there are several meanings similar to one particular } \\
\text { meaning that serves as primum analogatum }\end{array}$ \\
\hline equivocity & $\begin{array}{l}\text { there are different meanings each of which applies to } \\
\text { one singular field only }\end{array}$ \\
\hline
\end{tabular}

The first option is: there is only one meaning of the term "information", it means the same regardless of the field of application. This option is called synonymity, because the terms are synonyms.

The second option is: there are several meanings of the term "information", they are similar to a particular meaning, which serves as standard of comparison. This option is called analogy, analogical reasoning, because the terms are analogies.

The third option is: there are several meanings of the term "information", all of which are different from each other. This option is called equivocity, because the terms are equivocations.

No option, actually, meets demands for scientificity. Synonymity does not meet them, because information in one domain would not differ from information in a different domain a premiss which has long been contested. Analogical reasoning does not meet them either, because there is no agreement on the primum analogatum, the standard of comparison. Nor does equivocity meet them, because the babel of languages which are not communicable would mean the end of scientific enterprise at all.

Does this mean that we are stuck and that there is no solution to the trilemma?

No. The three options Capurro's trilemma is offering are tantamount to exactly three well- 
definable ways of thinking - to, so to say, the black, the white, and the black-and-white way of thinking, that is, the reductive, the projective, and the disjunctive way. But there is a fourth way of thinking - the integrative one (see Table 2).

Table 2 : Ways of thinking

\begin{tabular}{|l|l|}
\hline ways of thinking & $\begin{array}{l}\text { relationship between lower and higher } \\
\text { complexity }\end{array}$ \\
\hline reductionism & reduces higher complexity to lower complexity \\
\hline projectivism & projects higher complexity onto lower complexity \\
\hline disjunctivism & disjoins higher complexity from lower complexity \\
\hline integrativism & $\begin{array}{l}\text { integrates lower complexity into higher } \\
\text { complexity which it differentiates from the former }\end{array}$ \\
\hline
\end{tabular}

In detail. What is a way of thinking? A way of thinking is the way how identity and difference are thought to relate to each other. Relating identity and difference may be presumed to be the most basic function of thinking. That is, practical problems that come to thought, entities that are investigated, phenomena that have to be cognised, may be identical in certain respects but may differ from each other in other respects.

Regarding identity and difference, given complexity, that is, provided that what differs is more complex than that from which it differs, but, by the same token, instaurates an integrated whole, the question arises as to how the simple does relate to the complex, that is, how less complex problems or objects or phenomena do relate to more complex ones.

The first way of thinking, in terms of ideal types, establishes identity by eliminating the difference for the benefit of the less complex side of the difference and at the cost of the more complex side; it reduces "higher complexity" to "lower complexity"; this is known as reductionism. Reductionism is still the main stream of natural science.

The counterpart of the reductive way of thinking is what might be called projective. Projective thinking also establishes identity by eliminating the difference, albeit for the benefit of the more complex side of the difference and at the cost of the less complex side; it takes the "higher" level of complexity as its point of departure and extrapolates or projects from there to the "lower" level of complexity. It overestimates the role of the whole and belittles the role of the parts. This is one trait of humanities.

Both the reductive and the projective way of thinking yield unity without diversity.

To go on, there is a third way opposed to both of the others in that it eliminates identity by establishing the difference for the sake of each manifestation of complexity in its own right; it abandons all relationships between all of them by treating them as disjunctive; it dissociates one from the other, it dichotomises and yields dualism (or pluralism) in the sense of diversity without unity. Let's call it disjunctivism. The often bemoaned cleft between the so-called two cultures of hard science and soft science (humanities) is the most striking example for this way of thinking. In fact, this is a description of the state of the scientific adventure as a multiplicity of monodisciplinary approaches that are alien and deaf towards each other.

You can easily see that the options of synonymity, analogy and equivocity are reductive, projective and disjunctive respectively.

Either you have unity without diversity (in the first and second case) or you have diversity without unity (in the third case). What is needed, however, is "unitas multiplex" as French philosopher and sociologist Edgar Morin calls it $(1999,25)$, understanding unityin-diversity and diversity-in-unity, unitythrough-diversity: "It means understanding disjunctive, reductive thought by exercising thought that distinguishes and connects. It does not mean giving up knowledge of the parts for knowledge of the whole, or giving up analysis for synthesis, it means conjugating them. This is the challenge of complexity which ineluctably confronts us as our planetary era advances and evolves" $(1999,19)$. This is a way of thinking that establishes identity as well as difference favouring neither of the manifestations of complexity; it establishes identity in line with the difference; it integrates both sides of the difference (yielding unity) and it differentiates identity (yielding 
diversity); it is a way of thinking that is based upon integration and differentiation; it is opposed to both dissociation and unification and yields unity and diversity in one. It integrates "lower" and "higher complexity" by establishing a dialectical relationship between them.

This integrativism opposes reductionism and projectivism as well as disjunctivism.

The unity-through-diversity principle is itself a kind of dialectical sublation of unification and dissociation, of reductionism and projectivism and disjunctivism. A dialectical sublation eliminates the dominant role of the preceding quality rather than the quality itself. This quality is kept, that is, continued, but it is continued under the dominance of a new quality and is therefore - as Hegel put it lifted onto a next level. All of that holds for the unity-through-diversity thinking with regard to the fallacious ways of thinking. Reductionism, projectivism as well as disjunctivism are not totally negated but taken cum grano salis. Each of them has an aspect of overexaggeration that has to be abolished but, by the same token, it has an aspect that is right once the onesidedness is removed. Doing justice to these aspects is carried out through the novel integrative view - in such a way unity is established among the diverse confligating views.

To sum up, reduction, projection or duality are justified within certain boundaries and when taking into account the legitimate claims of each other. This is the integrative way of thinking a UTI has to carry out.

\section{Is a Unified Theory of Information (UTI) similar to a Grand Unified Theory (GUT) or a Theory Of Every- thing (TOE)?}

Yes and no.

Yes, insofar as both the UTI and the GUT/TOE aim at giving a bigger picture. The UTI seeks for understanding different manifestations of information processes in the universe just as the GUT/TOE tries to find a common denominator for the four fundamental interactions/forces gravitation, electromagnetism, the weak and the strong (quantum chromodynamics) interaction/force.
But no, if the GUT/TOE is meant as a pure physical theory for the explanation of physical phenomena only or as a physical theory which gives final explanations of phenomena other than physical in the universe. A UTI is not a pure physical theory and information not a pure physical phenomenon (like interaction/force) - which Carles Seife (2007) insinuates. Furthermore, the properties of information extending -beyond physicality- to living beings and social human life cannot be explained in terms of mere physics.

A UTI is not a physicalistic and hence reductionistic theory which yields a world formula that is said to allow for explanations and predictions by subsumption under a general and hence abstract construct.

A world formula does not prove feasible, and a unified concept of information is not a world formula.

What a UTI searches for is a concept as abstract as necessary but as concrete as possible at the same time.

The more abstract a concept is, the poorer it is by intension and the larger by extension. The more concrete a concept, the richer its intension and the smaller its extension.

On the one hand, the concept shall theorise what all information processes have in common but it shall not reduce to an abstract formalism that can subsume every case under a meaningless meaning.

On the other hand, it shall cover each individual information process that may empirically be found but not hypostatise its unique particularities into a concretistic notion.

That's the real challenge. We need a concept that is flexible enough to balance the universal and the particular, to do justice to both of them, to relate them so as to render the universal in need as well as capable of being completed by the particular and, in turn, embed the particular in the universal (see Hofkirchner 2004). Reductionistic unification would reduce the particular to the universal by stating "The Particular is (nothing but) Universal" and assuming that the universal is the necessary as well as sufficient condition for the particular. This is true of all kinds of subsumption. They overlook what goes beyond that which subsumes. Unification by projection would project the particular onto the universal 
and postulate "The Universal is (nothing but) Particular", thereby meaning that the particular is not only necessary but also sufficient to yield the universal. This holds for those illusions that extend what is in common to a realm where it is not. The disjunctive way of thinking would dissociate the particular from the universal by presuming "The Particular and the Universal are Disjoint" and would in doing so insinuate that both notions contradict each other. This leads to letting the particular fall apart, since there is no unifying bond. Either of these three ways of thinking is onesided because by relying on the formal-logical figure of necessary and sufficient conditions or of contradiction it focusses on the mutual dependence of the sides or on being opposites and does not comprise the full range of what is characteristic of any dialectical relation.

It is only the fourth way of thinking that integrates as well as differentiates the particular and universal. This point of view may be formulated "The Particular Sublates the Universal" - "sublation" in the threefold Hegelian sense denoting suspending, saving and elevating altogether:

- the particular suspends the universal; being the opposite of the universal, the particular contradicts the universal and transcends it;
- the particular saves the universal; the particular depends on the universal, the latter being the necessary, but not sufficient condition for the particular, the particular is based upon the universal;

- the particular elevates the universal to another level; in an asymmetrical effort, the particular turns the universal, as a consequence, from an abstract universal into a concrete-universal.

The concrete-universal is the unity that overarches the diversity of the particular. Aristotle paved the way for a dialectics of the universal and the particular by establishing specification hierarchies via genus proximum and differentia specifica. The whole tree can be considered to represent the concreteuniversal, and each ramification to specify one particular instantiation of the universal by making the abstract concrete.

Specification hierarchies of being are the logical way of grasping the history and genesis of becoming (unity of being and becoming).

In that way, a UTI seeks a concreteuniversal concept of information rather than an abstract one.

\section{References}

Brockman, J. (1995). The Third Culture. New York: Simon and Schuster.

Bunge, M. (1980). The Mind-Body Problem. Oxford: Pergamon Press.

Capurro, R., Fleissner, P., Hofkirchner, W. (1997). Is a Unified Theory of Information Feasible?. World Futures, 49(3-4) \& 50(1-4), 213-234.

Heylighen, F. (2007). Accelerating Socio-Technological Evolution. In G. Modelski, T. Devezas, \& W. Thompson (Eds.), Globalization as an Evolutionary Process, London: Routledge, 286-335.

Hofkirchner, W. (1999). The Quest for a Unified Theory of Information. Amsterdam: Gordon and Breach.

Hofkirchner, W. (2002). Projekt Eine Welt: Kognition - Kommunikation - Kooperation. Versuch über die Selbstorganisation der Informationsgesellschaft. Münster: LitVerlag.

Hofkirchner, W. (2004). Unity Through Diversity. Dialectics - Systems Thinking - Semiotics. Trans, 15(1), retrieved from http://www.inst.at/trans/15Nr/01_2/hofkirchner15.htm. 
Hofkirchner, W. Fuchs, C., Klauninger, B. (2005). Informational Universe. A praxeo-onto-epistemological Approach. In E. Martikainen (Ed.), Human Approaches to the Universe, Interdisciplinary Studies, Helsinki: Academic Bookstore, 7594.

Hofkirchner, W., Stockinger, G. (2003). Towards a Unified Theory of Information. 404nOtFOund, 1(24), retrieved from http://www.facom.ufba.br/ciberpesquisa/404nOtF0und/404_24.htm.

Küppers, B.-O.. (2000). Die Strukturwissenschaften als Bindeglied zwischen Natur- und Geisteswissenschaften. In B.-O. Küppers (Ed.), Die Einheit der Wirklichkeit, München: Wilhelm Fink, 89-105.

Morin, E. (1999). Seven Complex Lessons in Education for the Future. Paris: UNESCO.

Popper, K.R., Eccles, J.C. (1977). The self and its brain. Berlin: Springer.

Saxe, J.G. (2003). The Blind Men and the Elephant. Retrived on 2009/05/22 from: <http://www.cs.rice.edu/ ssiyer/minstrels/poems/1179.html> [There are several versions of this traditional Indian tale, e.g.: Wang, R. (1995). Retrived from: <http://www.cs.princeton.edu/ rywang/berkeley/258/parable.html>]

Seife, C. (2007). Decoding the universe. How the new science of information is explaining everything in the cosmos, from our brains to black holes. London: Penguin Books.

Shannon, C.E. (1948). A Mathematical Theory of Communication. The Bell System Technical Journal, 27(July, October), 379-423, 623-656.

Snow, P.C. (1998). The Two Cultures. A Second Look. Cambridge: Cambridge University Press.

Wilson, E.O. (1998). Consilience: The Unity of Knowledge. New York: Knopf.

Windelband, W. (1894). Geschichte und Naturwissenschaft. Retrieved from http://www.fhaugsburg.de/ harsch/germanica/Chronologie/19Jh/Windelband/win_rede.html

\begin{abstract}
About the Author
Wolfgang Hofkirchner

(born 1953 in Vienna; Dr.phil. in Political Science and Psychology) is Professor for Internet \& Society at the ICT\&S Center, Paris-Lodron-University of Salzburg since 2004 and Associate Professor for Technology Assessment at the Institute for Design and Technology Assessment, Vienna University of Technology since 2001. Specialised in Science-TechnologySociety, current focus on Information Society Theory and Foundations of Information Science.

He published over 100 contributions in the field of Information Science, including 18 monographs or edited volumes. Member to, and serving on the board of, numerous scientific societies, member of 3 editorial boards and editor of TripleC and a book series. Some relevant publications: "Web 3.0 Philosophy: Internet in the perspective of a unified theory of information" (In Practychna Filosofiya 1(13), 2009); "Toward a New Science of Information" (In Proceedings CODATA 2006, Data Science Journal, Vol. 6, 7 April 2007, co-authored with Doucette, Bichler \& Raffl); "Biological Information. Sign Processes in Living Systems" (In Journal of Biosemiotics 2, Vol. 1, 2005, co-authored with Ellersdorfer); "A New Way of Thinking and a New World View. On the Philosophy of Self-Organisation I" (In Cybernetics \& Human Knowing Vol. 11/No. 1, 2004); The Quest for a Unified Theory of Information. Proceedings of the Second Conference on the Foundations of Information Science (Amsterdam: Gordon\&Breach, 1999).
\end{abstract}

